\title{
Buchbesprechung
}

\author{
A. Benauwt, A. Lorette
}

\section{Manuel d'Orthodontie pour omnipraticiens}

1977. 119 S., 93 Abb. (Julien Prélat, Paris).

Die beiden Autoren, ehemalige Assistenten der Fakultät von Paris, wollen mit ihren Ausführungen dem Allgemeinpraktiker Hinweise auf die kieferorthopädische Tätigkeit geben.

Das Buch gliedert sich daher auch in zahlreiche Kapitel, in denen die Entwicklung der Orthodontie, das Fernröntgenbild und das Wachstum sowie die besondere kieferorthopädische Diagnostik als Grundlage der Behandlungsplanung mit ihren wichtigsten Aspekten abgehandelt werden. Es folgt eine Beschreibung der verschiedenen Typen von kieferorthopädischen Behelfen, einschließlich der Mittel der Retention und ihrer Herstellung im Laboratorium. Ein Kapitel, in dem zu verschiedenen Einzelfragen, wie Hygiene und Orthodontie, Nichtanlage von Zähnen, Verlagerung von Zähnen und ihre Einordnung und dergleichen mehr, Stellung genommen wird, beschließt außer einer kurzen Bibliographie das interessante Buch.

Die Entwicklung der Kiefercrihopädie und ihre Bedeutung für die zahnärztliche Praxis wird in dem vorliegenden Buch in überzeugender Weise dargelegt, denn auch der zahnärztliche Allgemeinpraktiker findet darin eine große Zahl von wichtigen Hinweisen auf kieferorthopädische und orthodontische Möglichkeiten, die für seine Patienten von besonderer Bedeutung sein dürften, auch wenn er sie selbst nicht ohne weiteres anwenden wird. Für uns ist es besonders interessant, aus dem Buch zu ersehen, in welchem Umfange Kieferorthopädie auch in der Allgemeinpraxis in Frankreich inzwischen Eingang gefunden hat .

Die Ausstattung des Buches ist ausgezeichnet, und die zahlreichen, guten Abbildungen sind eine hilfreiche Erläuterung des Textes.

E. Hausser, Hamburg 